\title{
INFINITY: AN INTERDISCIPLINARY ACCESS KEY TO PHILOSOPHICAL EDUCATION THROUGH MATHEMATICS
}

\author{
Paolo Bussotti \\ The Alexander von Humboldt Foundation, Berlin, Germany \\ E-mail: paolobussotti66@gmail.com
}

In some previous contributions of mine written for Scientia Educologica's journals (Bussotti 2012; Bussotti, 2013; Bussotti, 2014) I dealt with the possible use of history of mathematics and science inside mathematics and science education. There is an abundant literature on this subject and I only tried to offer some ideas on possible educative itineraries in which history of mathematics and science could play a role. I had no claim to supply elements for a general theory on the relations history of mathematics-mathematics education and history of science-science education. In this editorial, I would like to deal with a possible interdisciplinary link between philosophical education and mathematics. This link is given by the infinity. The following considerations are valid for all those countries in which some high schools exist where philosophy is taught and, in general, for every course at a philosophical faculty in which the problem of the infinity is faced. Furthermore, they can also be useful in the teaching of mathematics at the high school when the concepts of infinity and infinitesimal (typically while dealing with calculus) are introduced.

The author who represents an almost natural link between philosophy and mathematics, as far as the problem of the infinity is concerned, is Georg Cantor (1845-1918) and his set theory. Set theory is - in general - taught in the courses of mathematical logic at the faculties of philosophy and mathematics or in the courses of history of mathematics at the faculty of mathematics. To this subject is dedicated an almost boundless and profound literature. However, I am here proposing a wider use of: 1) mathematical concepts; 2) specifically, Cantor's works to introduce and make it clearer the theme of infinity.

Let us suppose that a professor or a teacher is explaining San Augustin's (354-430) philosophy. In the $12^{\text {th }}$ book, $18^{\text {th }}$ chapter of De Civitate Dei, while dealing with transcendence and creation of time, Augustin claims, there is no doubt, that God knows the whole of number and the whole of the number is infinite. Augustin develops this interesting reasoning: given a number, it is possible to add a unity, it is possible to double this number and to develop a further series of operations. Each number determined in this manner is finite and different from another number, but the whole of number is infinite.

Other context: Baruch Spinoza's (1632-1677) philosophy and, in particular his Etica more geometrico demonstrata. Spinoza claims that, beyond the absolute infinity, who is Godthe substance, further infinities exist, in particular the modi and the attributes of God. These are infinite quantities. According to Spinoza, they cannot be expressed by a number. However, they are existing infinities, which cannot be identified with God.

Further example: Hegel (1770-1831) in numerous parts of his works uses the expression "bad infinity" (das Schlecht-Unendliche). In particular, in a note inside Science of Logic (Wissenschaft der Logik) he clarifies that bad infinity is a never closed process consisting in continuously overcoming a given limit, never reaching a conclusion. While the good infinity is a given infinity involving no process. According to Hegel, the notions of space and time used in 
Paolo BUSSOTTI. Infinity: An Interdisciplinary Access Key to Philosophical Education through Mathematics

PROBLEMS

OF EDUCATION

IN THE $21^{\text {st }}$ CENTURY Volume 60, 2014

science are bad infinities, whereas the Concept and the Spirit who, at the end of a finite process, becomes conscious of himself are good infinites (as a matter of fact, the same and only good infinity).

The three examples could concern part of a course in history of philosophy or a course on the philosophical concept of infinity or they could be part of a monographic course on one of the three authors. The examples could be multiplied, including the numerous philosophers who dealt with the problem of infinity.

Considering that in the high schools, the theme of infinity is not, in general, dealt with in the mathematics courses (in the courses concerning calculus, of course, one speaks of limits and of infinity, but, in general, without giving a definition of this concept), a student can find some difficulties:

- Augustin claims that, given a number, it is possible to add always a further number. This is obvious. Hence the numbers are infinite, in the sense that, given a number, I can add 1 and to find another number. But, it seems that Augustin is referring to another concept: the whole of number, but what does this mean?

- Hegel speaks of infinity as a process, which is the bad infinity, and another infinity, which is the good infinity, but what is the difference?

To make these initial differences clear, mathematics can play a fundamental role. It is about the difference between potential and actual infinity. Here - at the high school - it is possible to think of a collaboration between the teacher of philosophy and of mathematics, and at the university between the professors of philosophy and those of logic or history of mathematics. Let us start from Augustin: when, given a number $n$, we add 1, reaching the number $n+1$, every number is finite, however the process is endless, we can continue to add 1 . In this manner we obtain the series of the natural numbers: each number is finite, the process of addition can be continued at every step, hence we say that this series is potentially infinite because, given a number of the series, it is always possible to find a number which is greater than the given one. The same notion of potential infinity is involved in the concept of limit in mathematical analysis. For example, when we say that, this means that, given any $M$ (which is supposed "big"), starting from a given value of $x$ (which will be "near" 0 ) all the values of will be greater than $M$. However, this does not mean that in $x=0$, the function gets the value $\infty$, simply because in $x=0$, such function does not exist. The symbol $\infty$ indicates hence a potential infinity.

Now, let us consider the question from a different perspective. If we take into account all the natural numbers or all the numbers which belong to a segment of the real straight line, for example the interval $[0,1]$, and we ask, how many are these numbers? We have to answer that they are infinite. Nevertheless, this infinity is completely different from the potential infinity, because, in this second case, no process is involved: the natural numbers or the numbers belonging to the interval $[0,1]$ are a given whole - nowadays, exactly following Cantor's language - we speak of set. The number of elements of the two considered sets is infinite. This infinity is called actual infinity and it is an infinity in a proper sense, not a finite quantity which can become greater than a given finite quantity. This is the kind of infinity to which Augustin was referring when he spoke of the whole of number, to which Hegel was referring when he spoke of good infinity, whereas the potential infinity is the bad infinity, according to Hegel. Mathematics is hence fundamental to provide the students with a clear distinction between the two conceptions of infinity, which plays such an important role in the development of philosophy. Once the students have caught this difference by means of mathematics, it is, of course, necessary to come back to philosophy and to insert these concepts in their specific philosophical context. 
However, if we read Spinoza, things appear more complicated than one student in phi-

PROBLEMS

OF EDUCATION

IN THE $21^{\text {st }}$ CENTURY

Volume 60,2014

losophy could think, because Spinoza speaks of different kinds of actual infinities. What does this mean? If, to answer the previous questions, some general mathematical considerations were sufficient, here it is appropriate to make reference directly to Cantor. Once again, an interdisciplinary itinerary philosophy-mathematics can be organized.

In 1878 (see Cantor 1878, 1932) Cantor defined two sets $M$ and $N$ equivalent if a biunivocal correspondence between the elements of the two sets exists. This definition is not interesting if one limits to consider finite sets, but it becomes interesting, if we take into account sets with an infinite number of elements. In 1874 (see Cantor 1874, 1932) Cantor had been able to prove that all the algebraic numbers, that is, all the numbers which are solution of algebraic equations with integral coefficients (the rational numbers belong, for example, to the set of the algebraic numbers), are equivalent to the set of the natural numbers. While the set of the transcendent numbers - which are not the solution of such equations - is not equivalent to the set of the integer numbers: it is bigger. This means - obviously - that the set of all real numbers is not equivalent to the set of the natural numbers; it is equivalent to that of the transcendent numbers. Thence, although this is not intuitive:

1) the sets of the natural numbers, of the rational numbers and of the algebraic numbers are all equivalent, belong to the same class of actually infinite sets.

\section{Table 1. A "visual" demonstration that the squares are as numerous as all the} natural numbers.

\begin{tabular}{llllll}
\hline 1 & 2 & 3 & 4 & $\ldots$ & $\begin{array}{l}\text { Although from an intuitive point of view, the set of the natural numbers } \\
\text { is more numerous than that of the squares because there are numbers } \\
\text { which are not squares, this is not true according to Cantor's definition of } \\
\text { equivalent sets. For, a biunivocal correspondence exists between the two } \\
\text { sets, as the figure on the left shows. }\end{array}$ \\
1 & 4 & $\uparrow$ & $\uparrow$ & $\uparrow$ &
\end{tabular}

Whereas:

2) the set of the transcendent numbers, of the real numbers, but also of the real numbers included in a segment of straight line as $[0,1]$ belong to a different class. They are "bigger" than the set of the natural numbers. 
PROBLEMS

OF EDUCATION

IN THE $21^{\text {st }}$ CENTURY

Volume 60,2014

Table 2. Easy demonstration that set of the real numbers of the segment $[0,1]$ is not equivalent to the set of the natural numbers.

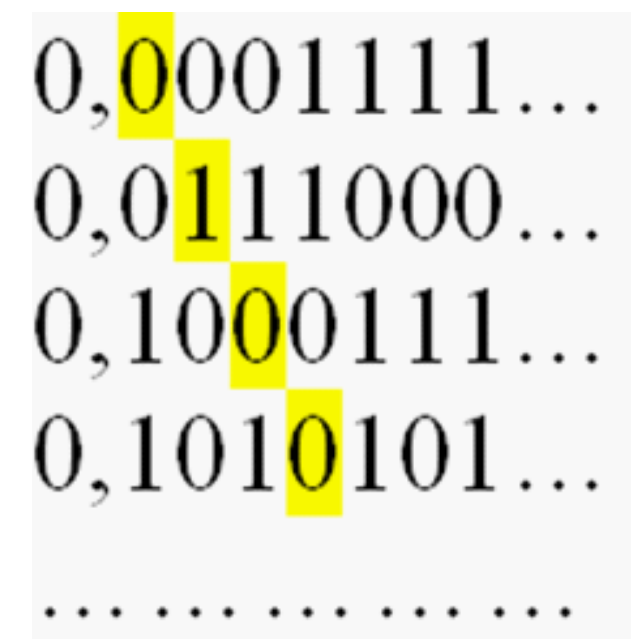

Let consider the numbers belonging to the segment $[0,1]$ in which only the digits 1 and 0 appear. Let us suppose that they are in a biunivocal correspondence with the set of natural numbers. Then it is possible to give them an order in which one number is the first, one is the second and so on. In this series all the mentioned numbers should appear. Let us suppose that the series is as in the figure. Now let us replace the digits indicated in yellow - namely the digits which appear in the first line first column after the comma for the first number; the second line and the second column after the comma in the second number and so on - so that every 0 is replaced by 1 and vice versa. It is evident that - by means of this procedure - one gets a number belonging to the interval $[0,1]$ which does not belong to our series. This proves that the numbers belonging to $[0,1]$ cannot be ordered in a series, hence this set is not equivalent to that of the natural numbers: it is bigger. This proof - which can be found in every handbook of set theory is an adaptation of Cantor's original.

It is also legitimate to wonder whether other classes of actually infinite sets exist beyond the mentioned two. The answer is affirmative and, as Cantor showed (Cantor 1895-1897, 1932, 1955), it is easy to produce them. Let us consider a set $N$ composed of a finite number of elements - for example by a number $n$ of elements -, then it is not difficult to prove that the number of elements of the set of all the subsets of $N$, also called power of $N$ - let us indicate it by $P(N)$ - is . This relation holds also if $N$ is an infinite set. Cantor proved that no biunivocal relation exists between the infinite set $N$ and $P(N)$; but $P(N)>N$. In this manner, it is evident that we get an infinite series of actually infinite sets, by considering $N, P(N), P(P(N)), \ldots$, where $N$ is an infinite set.

Table 3. The concept of set of all the subsets, or power, of a given set.

I provide an example to clarify the relation between a set $N$ and the set of all its subsets - or power - of $N, P(N)$. Be $N=$. By convention, the empty set belongs to every set. Hence the power of

$N$ is: $P(N)=$. The numbers of elements of $P(N)$ - elements which are sets - is exactly, as the general theorem claims.

By this explanation a student who is interested in philosophy, without a specific interest in logic or mathematics, can get a clear idea of what an actually infinite quantity is and, for sure, he will meet few difficulties to understand these concepts from a philosophical standpoint. While, without any mathematical introduction, I think these concepts might remain rather vague and obscure. Mathematics can in this case help philosophy education. Mine is only an example as I am convinced that numerous other interdisciplinary itineraries mathematics-philosophy could be traced. I provided this example not by chance. For instance, I have shown how to explain, by means of mathematics, the concept of actual infinity in a context of philosophical education. Another interesting educational itinerary could be something opposite: how philosophy influences the concept of actual infinity in mathematics. The connection Spinoza-Cantor outlined in these pages is not fortuitous as Cantor was deeply influenced by Spinoza and it is possible to prove that there are profound connections between their conceptions of infinity (Bussotti, Tapp, 2009). 
The way of an interdisciplinary education is open and fruitful, but it is necessary to cross it by means of concrete and applicable educative situations and examples rather than by abstract theoretical considerations.

\section{References}

Bussotti, P. (2012). History and didactics of mathematics: A problematic relation. Some considerations based on Federigo Enriques's ideas. Problems of Education in the $21^{\text {st }}$ Century, 48, 5-9.

Bussotti, P. (2013). A possible role of history of mathematics and science in mathematics and science education. Journal of Baltic Science Education, 12 (6), 712-715.

Bussotti, P. (2014). The scientific revolution of the $17^{\text {th }}$ century. The aspects connected to physics and astronomy: An educational itinerary in seven lessons. Problems of Education in the $21^{\text {st }}$ Century, $58,5-12$

Bussotti, P., Tapp, C. (2009). The influence of Spinoza's concept of infinity on Cantor's set theory. Studies in History and Philosophy of Science, 40, 25-35.

Cantor, G. (1932). Gesammelte Abhandlungen mathematischen und philosophischen Inhalts (E. Zermelo, Ed.), Berlin: Springer.

Cantor, G. (1874, 1932). Über eine Eigenschafte des Inbegriffs aller reellen algebraische Zahlen. In Cantor, 1932, 115-118. Freely available at: http://www.cs.elte.hu/ badam/matbsc/11o/cantor1874. pdf.

Cantor, G. (1878, 1932). Ein Beitrag zu Mannigfaltigkeitslehre. In Cantor 1932, 119-138.

Cantor, G. (1895-1895, 1932, 1915-55). Beiträge zur Begründung der transfiniten Mengenlehre. In Cantor 1932, 282-355. Translated into English in 1915 (second edition1955) as Contributions to the Founding of the Theory of transfinite Numbers. New York: Dover. Retrieved from https://archive. org/details/contributionstot003626mbp. 